ISSN 1997-342X (Online), ISSN 1991-8631 (Print)

Original Paper http://ajol.info/index.php/ijbcs http://indexmedicus.afro.who.int

\title{
Prévalence des souches d'entérobactéries productrices de bêta-lactamases à spectre élargi isolées au Togo et de leur sensibilité aux antibiotiques
}

\author{
Akouétévi Gérard TOUDJI ${ }^{1,3}$, Bouraïma DJERI ${ }^{1}$, Simplice Damintoti KAROU ${ }^{1 *}$, \\ Ségla TIGOSSOU ${ }^{2,3}$, Yaovi AMEYAPOH ${ }^{1}$ et Comlan de SOUZA $^{1}$ \\ ${ }^{I}$ Laboratoire de Pharmacopée du Centre de Recherche et de Formation sur les Plantes Médicinales \\ (CERFOPLAM) de l'Ecole Supérieure de Techniques Biologiques et Alimentaires de l'Université de Lomé \\ (ESTBA), Lomé, Togo. \\ ${ }^{2}$ Laboratoire de microbiologie du CHU-Sylvanus Olympio, Lomé, Togo. \\ ${ }^{3}$ Laboratoire de microbiologie de la Polyclinelle Wossinu et Gbogbo, Lomé, Togo, \\ *Auteur correspondant ; E-mail : simplicekarou@hotmail.com
}

\section{RESUME}

La dissémination des bactéries multi-résistantes aux antibiotiques contribue à la morbidité et la mortalité dues aux maladies infectieuses. Le but de cette étude était de déterminer la prévalence des souches d'entérobactéries productrices de bêta-lactamases à spectre élargi (EBLSE) et d'évaluer leur sensibilité aux antibiotiques. Au total 1377 souches ont été isolées au CHU Sylvanus Olympio et à la Polyclinelle Wossinu-Gbogbo entre 2009 et 2011. Les tests de sensibilité selon le Comité d'Antibiogramme de la Société Française de Microbiologie et de synergie en double disque pour détecter les BLSE ont été réalisés. Au total 309 souches ont produit les BLSE soit une prévalence de 22,44\% (309/1377). Escherichia coli a été plus fréquent (51,13\%), suivi de Klebsiella spp. (30,10\%), $(\mathrm{p}<0,01)$. Ces germes ont été isolés des pus à $47,90 \%$ et des urines à $40,78 \%$. Les résistances avec $E$. coli ont été respectivement de $0,6 \%$ et $1,3 \%$ pour l'imipénème et la colistine. Concernant Klebsiella spp., ces taux ont été de $3,7 \%$, et de 1,27\% respectivement. La prévalence des EBLSE a été de 22,44\%. E. coli, Klebsiella spp. ont été les plus impliqués avec une forte résistance à la majorité des antibiotiques. D'autres études et des alternatives d'antibiothérapie devraient être envisagées.

(C) 2017 International Formulae Group. All rights reserved.

Mots clés : Souches bactériennes, multirésistantes, bêta-lactamase à spectre élargi, antibiotiques.

\section{Prevalence of extended spectrum beta-lactamases producing enterobacteria and their susceptibility to antibiotics in Lome, Togo}

\begin{abstract}
The dissemination of antibiotic-resistant bacteria contributes to morbidity and mortality from infectious diseases. The aim of this study was to investigate the prevalence of extended spectrum beta-lactamase producing enterobacteria (ESBLE) strains and assess their susceptibility to antibiotics. A total of 1377 strains were isolated at the CHU Sylvanus Olympio and the Polyclinelle Wossinu-Gbogbo between 2009 and 2011. The susceptibility test according to the Antibiogram Committee of the French Microbiology Society and
\end{abstract}


double disk synergy test for the detection of ESBL was carried out. A total of 309 strains produced ESBLs, a prevalence of $22.44 \%$ (309/1377). E. coli led with $51.13 \%$, followed by Klebsiella spp. $(30.10 \%)$, (p $<0.01$, Fisher's law), most isolated from pus (47.90\%), urine (40.78\%). E. coli resistance was $0.6 \%$ and $1.3 \%$, respectively to imipenem and colistin. Concerning Klebsiella spp., these rates were $3.7 \%$ for imipenem and $1.27 \%$ for colistin. The prevalence of EBLSE was $22.44 \%$. E. coli, and Klebsiella spp. were most implicated with strong resistance to the majority of antibiotics. Other studies and alternatives to antibiotic therapy should be considered.

(C) 2017 International Formulae Group. All rights reserved.

Keywords: Bacterial strains, multiresistance, extended spectrum beta-lactamase, antibiotics.

\section{INTRODUCTION}

L'émergence et la dissémination des bactéries ayant acquis des résistances aux antibiotiques, constituent un énorme problème de santé publique (Bradford, 2001; Ahoyo et al., 2007; Rastogi et al., 2012). La conséquence directe de cette antibiorésistance est l'augmentation de la morbidité et de la mortalité dues aux maladies infectieuses (Hailaji et al., 2016). Une première approche dans la lutte contre les antibiorésistances implique les études de prévalence des souches multi résistantes circulant dans les milieux hospitaliers ainsi que leur sensibilité aux antibiotiques. Ainsi, les études menées un peu partout dans le monde ont surtout incriminé les cocci à Gram positif (Quincampoix et Mainardi, 2001) et les bacilles à Gram négatif, notamment les entérobactéries (Kader et al., 2004; Luzzaro et al., 2006). Ces dernières expriment un taux élevé de résistance acquise à la majorité d'antibiotiques $\mathrm{du}$ groupe des bêta-lactamines par la production d'enzymes dénommées les bêtalactamases à spectre élargi (BLSE) inactivant les céphalosporines de première, de deuxième et de troisième génération (Paterson et Bonomo, 2005 Rawat et Nair, 2010). Les gènes qui codent pour ces enzymes sont portés par des plasmides et coexistent avec les gènes de résistance à d'autres antibiotiques d'où l'origine de la multi résistance des entérobactéries productrices de bêtalactamases à spectre élargi (EBLSE) (Spanu et al., 2002 ; Rawat et Nair, 2010). Les espèces les plus fréquemment isolées sont Escherichia coli et Klebsiella pneumoniae (Aminzadeh et al., 2008 ; Somily et al., 2014).
$\mathrm{Au}$ Cameroun, les recherches ont fait cas de 12\% d'Entérobactéries Sécrétrices de Bêta-Lactamases à Spectre élargi (EBLSE) dont $18,8 \%$ de $K$. pneumoniae et $14,3 \%$ d' $E$. coli. (Gangoué-Piéboji et al., 2005). Au Bénin, dans le département de Zou et Collines, 22\% les souches d' $E$. coli BLSE ont été isolées Ahoyo et al., 2007). Le Togo, à l'instar de ces pays, n'est sans doute pas épargné du problème, comme en témoignent les résultats rapportés par quelques auteurs (Dagnra et al., 2000 ; Karou et al., 2012). Cependant, aucune étude publiée ne s'est intéressée tout particulièrement aux BLSE. La présente étude a eu pour objectifs de déterminer la prévalence des entérobactéries productrices de bêta-lactamases à spectre élargi dans deux structures sanitaires de Lomé, et de tester leur sensibilité aux antibiotiques.

\section{MATERIEL ET METHODES \\ Isolement et identification des souches d'entérobactéries}

Les souches bactériennes ont été isolées au CHU Sylvanus Olympio et la Polyclinelle Wossinu-Gbogbode Lomé en suivant les méthodes en vigueur dans les deux centres. Les prélèvements vaginaux, d'urines et de pus ont été directement ensemencés sur le milieu gélosé EMB. Les prélèvements sanguins ont été mis dans des flacons d'hémoculture hémoline de la maison Bio Mérieux pendant au moins 3 jours pour noter la croissance bactérienne. Après la coloration de Gram, une subculture sur EMB est réalisée et l'identification des souches est faite par la galerie API 20E. (Bio Mérieux, France). 
Antibiogramme et détection de la production des bêta lactamases à spectre élargi

La sensibilité aux antibiotiques a été effectuée par les tests de diffusion en disque selon le Clinical and Laboratory Standard Institute et leur interprétation s'est faite suivant les recommandations du Comité d'Antibiogramme de la Société Française de Microbiologie (CA-SFM, 2004). Les critères de catégorisation ont été définis en sensible $(\mathrm{S})$, intermédiaire (I) et résistant $(\mathrm{R})$ pour chaque antibiotique utilisé. Vingt-quatre antibiotiques (amoxicilline, amoxicilline + acide clavulanique, carbénicilline, imipénème, céfalotine, céfoxitine, céfotaxime, ceftazidime, ceftriaxone, gentamicine, amikacine, nétilmicine, kanamycine, chloramphénicol, colistine, acide nalidixique, ciprofloxacine, péfloxacine, lévofloxacin, ofloxacine, norfloxacine, doxycycline, tétracycline, triméthoprime + sulfaméthoxazole) ont été testés.

La production de bêta-lactamases à spectre élargi a été mise en évidence par la technique de synergie en double disque décrite par Olonitola et al. (2007). Le disque d'amoxicilline+acide clavulanique (AMC) est placé entre les disques ceftazidime, de ceftriazone ou de céfotaxime (céphalosporine de troisième génération $\mathrm{C} 3 \mathrm{G}$ ) à une distance de 2 à 3 centimètres de centre à centre sur une boite de gélose Mueller Hinton. Après une incubation de 18 à 24 heures, il apparaît une zone sans culture en dôme entre les disques de l'AMC et ceux des $\mathrm{C} 3 \mathrm{G}$ dite en «bouchon de champagne».

\section{Cinétique de l'activité bactéricide des antibiotiques et de leurs associations}

La cinétique bactérienne a été étudiée avec deux antibiotiques arbitrairement choisis. Il s'est agi de l'Imipénème et l'Amikacine et de leur association. Les solutions d'antibiotiques: quatre disques de chaque antibiotique (imipénème à $10 \mu \mathrm{g}$ et l'amikacine à $30 \mu \mathrm{g}$ ) ont été mis dans cinq millilitres de bouillon $\mathrm{MH}$. Le bouillon $\mathrm{MH}$ contenant l'IPM est à $10 \mu \mathrm{g} / \mathrm{L}$ et celui de l'AN est à $30 \mu \mathrm{g} / \mathrm{l}$. Ces solutions d'antibiotiques ont été diluées à volonté et mis en contact avec l'inoculum bactérien. Ces tests ont été effectués dans des microplaques de 96 puits avec une culture en phase exponentielle des souches de Klebsiella pneumoniae. Des puits sans antibiotiques ont servi de control de croissance des bactéries. Les plaques ont été incubées à $37{ }^{\circ} \mathrm{C}$ sous agitation continue. La numération bactérienne s'est faite à $0,15,30$, 60 , 90 et 120 minutes d'incubation par prélèvement de $10 \mu 1$ de suspension suivi d'un ensemencement d'une boîte pétri incubée à 37 ${ }^{\circ} \mathrm{C}$ pendant 24 heures. Les courbes représentant le nombre de bactéries viables (exprimé en $\mathrm{Log}$ ) en fonction du temps en minutes de contact avec chaque antibiotique (IMP et AN) et leur association ont été tracées par GRAPH PAD PRISM6 afin d'apprécier le type d'interaction observée et le temps de bactéricidie.

\section{RESULTATS}

Prévalence des souches d'entérobactéries productrices de bêta-lactamases à spectre élargi

Cette étude a porté sur un total de 1377 souches d'entérobactéries. Le Tableau 1 montre les genres d'Enterobacteriaceae impliquées. Il s'est agi de Escherichia coli, Klebsiella spp, Enterobacter spp, Citrobacter spp, Proteus spp et E. coli Alkalescens-Dispar (E. coli $A-D)$. En terme de fréquence d'isolement, $E$. coli 48,29\% (665/1377) et Klebsiella spp. 24,25\% (334/1377) sont de loin des souches les plus fréquemment isolées soit $72,54 \%$ du nombre total d'isolat. Enterobacter spp et Proteus spp ont un taux d'isolement équivalent à $8 \%$. Le test de synergie en double disque a montré que 309 des 1377 souches isolées ont produit les BLSE soit un taux de 22,44\% (Tableau 2). Parmi ces souches, $E$. coli a été plus isolé à $52,75 \%$ avec $1,62 \%$ de la sous-espèce E. coli AlkalescensDispar. Ensuite Klebsiella spp. a suivi à un taux de 30,10\%. Enfin, les souches de Enterobacter spp. ont représenté $13,27 \%$ des isolats. Les trois genres d'entérobactéries réunis font 292 des 309 souches 
d'entérobactéries BLSE soit 96,12\%. Lorsque les résultats sont exprimés en prévalence au sein de chaque espèce, les Escherichia coli ont été toujours en tête à $39,89 \%$ avec jusqu'à $16,13 \%$ E. coli $A-D$. Ensuite, ont suivi Enterobacter spp à 35,34\% (41/116). Et enfin, Klebsiella spp. a été à $27,84 \%$ (93/334). La Figure 1 exprime le nombre de souches de EBLSE isolées par échantillon. Les pus ont rassemblé (148/309) des EBLSE isolées, soit $47,90 \%$ suivis des urines (126/309), soit $40,78 \%$.

La Figure 2 exprime la répartition des souches EBLSE isolées dans les échantillons traités. E. coli a été le plus fréquemment isolés des $\mathrm{PV}$, urines et pus à des taux respectifs de $63,64 \%, 57,94 \%$ et $47,97 \%$. Les souches de Klebsiella spp. ont constitué le second grand groupe d'isolat dans ces mêmes échantillons à des taux variables. Par contre, elles ont été plus isolées dans le prélèvement de sang à un taux de $41,67 \%$. Les souches Enterobacter spp. ont représenté le troisième groupe d'isolat dans tous les échantillons.

\section{Profil de résistance des Enterobacteriaceae BLSE isolées aux différents antibiotiques utilisés}

Le Tableau 3 illustre le profil de résistance des entérobactéries les plus représentées à savoir E. coli, Klebsiella spp., Enterobacter spp., Proteus spp. et E. coli A-D. L'ensemble des souches a été fortement résistante jusqu'à $100 \%$ aux beta-lactamines. L'Imipénème au contraire a totalement inhibé toutes les souches de Entérobacter spp, E. coli $A-D$ et de Proteus spp. tandis que les souches de E. coli et de Klebsiella spp. ont affiché une résistance respectivement à $0,68 \%$ et $3,7 \%$. Ce niveau de résistance exprimé par l'ensemble des souches étudiées a montré une différence significative par les valeurs $\mathrm{p}<0,05$ calculées selon la loi de Fisher avec quatre degré de liberté. Les souches de E. coli $A-D$ n'ont pas exprimé de résistance face aux aminosides du moins pour amikacine et la nétilmicine qui ont été testés. Envers ces mêmes antibiotiques, les Proteus spp ont montré une résistance respectivement de $30 \%$ et $40 \%$ mais ont vaincu la gentamicine et la kanamycine à $100 \%$. Chez E. coli, Klebsiella spp et Enterobacter spp, la résistance a paru stratifiée. Elle a été plus faible envers l'amikacine moins de 14\%; moins de $38 \%$ envers la nétilmicine, moins de $89 \%$ envers la kanamycine, et moins de $97 \%$ envers la gentamicine. Enterobacter spp. et Proteus spp. ont résisté à $100 \%$ au chloramphénicol. La colistine a fortement inhibé toutes les souches mais son action a été nulle sur Proteus spp. Les quinolones et les fluoroquinolones ont eu une activité fortement limitée sur ces souches BLSE de même que les sulfamides et les cyclines. La sensibilité des souches de E. coli et Klebsiella pneumoniae BLSE à la céfoxitine a été respectivement de à $83 \%$ et $81 \%$ dans le Tableau 4. Les diamètres d'inhibition mesurés autour du disque de céfoxitine ont donné une moyenne à $23,86 \mathrm{~mm}$ pour $E$. coli et à 21,69 $\mathrm{mm}$ pour $K$. pneumoniae. La médiane est la valeur de la distribution la plus représentée. Elle a été de $25 \mathrm{~mm}$ chez $E$. coli et de $22 \mathrm{~mm}$ pour $K$. pneumoniae. La différence entre les deux profils de sensibilité a été bien significative $(\mathrm{p}<0,05)$.

\section{Cinétique d'inhibition des antibiotiques vis- à-vis des souches de Klebsiella pneumoniae BLSE et non BLSE}

IPM et AN ont été très actifs sur les souches. Nous avons alors étudié leur effet sur la cinétique de la croissance bactérienne. La Figure 3 montre les courbes de la cinétique d'inhibition de Klebsiella pneumoniae non BLSE par les antibiotiques obtenues par le logarithme à base dix du nombre d'unité formant colonies par microlitre (UFC/ $\mu \mathrm{l})$ en fonction du temps. La courbe témoin à partir d'une culture sans inhibiteur a permis de comparer les densités des colonies par intervalle de temps régulier; la concentration bactérienne de départ était de $600 \mathrm{UFC} / \mu 1$. La bactéricidie a été observée très tôt à $15 \mathrm{mn}$ avec une réduction au dixième de la charge bactérienne obtenue avec l'AN et l'IPM-AN ; cette action a été totale après $30 \mathrm{mn}$. L'inhibition bactérienne a été par contre 
progressive avec l'IPM car environ le $1 / 10^{\text {ème }}$ de la densité a persisté après 2 heures de culture.

La cinétique de l'inhibition de la culture bactérienne est évaluée sur la Figure 4 avec les souches de Klebsiella pneumoniae BLSE. L'inoculum de départ est à 780 UFC/ $\mu$ l. Après $30 \mathrm{mn}$ d'incubation, la densité bactérienne a été réduite à $62,82 \%$ par l'AN et à 74,36\% par l'IPM. L'association IPM-AN a été plus active avec $5,26 \%$ de la population bactérienne résiduelle au bout de la même période. Dans cet essai, la combinaison des deux antibiotiques a eu un effet supérieur aux effets additionnés des deux inhibiteurs pris isolément. Nous pouvons parler de la synergie d'action des deux antibiotiques sur les souches de Klebsiella pneumoniae BLSE.

Tableau 1: Nombre et pourcentage des souches d'entérobactéries isolées.

\begin{tabular}{lcc}
\hline Souches isolées & Nombre de total & Pourcentage d'isolat \\
\hline Escherichia coli & 665 & 48,29 \\
Klebsiella spp. & 334 & 24,25 \\
Enterobacter spp & 116 & 8,42 \\
Citrobacter spp. & 21 & 1,52 \\
Proteus spp & 119 & 8,64 \\
E. coli $A-D$ & 31 & 2,25 \\
Autres entérobactéries & 91 & 6,61 \\
\hline
\end{tabular}

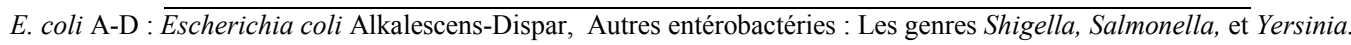

Tableau 2 : Prévalence des souches d'entérobactéries productrices de BLSE.

\begin{tabular}{lcc}
\hline Souches isolées & \multicolumn{2}{c}{ Souches BLSE } \\
\cline { 2 - 3 } & Nombre (\%) & \% de positivité BLSE) \\
\hline Escherichia coli $(\mathrm{n}=665)$ & $158(23,76)$ & 51,13 \\
Klebsiella spp. $(\mathrm{n}=334)$ & $93(27,84)$ & 30,1 \\
Enterobacter spp. $(\mathrm{n}=116)$ & $41(35,34)$ & 13,27 \\
Citrobacter spp. $(\mathrm{n}=21)$ & $3(14,29)$ & 0,97 \\
Proteus spp. $(\mathrm{n}=119)$ & $9(7,56)$ & 2,91 \\
E. coli $A-D(\mathrm{n}=31)$ & $5(16,13)$ & 1,62 \\
Autres Entérobactéries $(\mathrm{n}=91)$ & $0(0,00)$ & 0 \\
Total & 309 & 22,44 \\
\hline
\end{tabular}




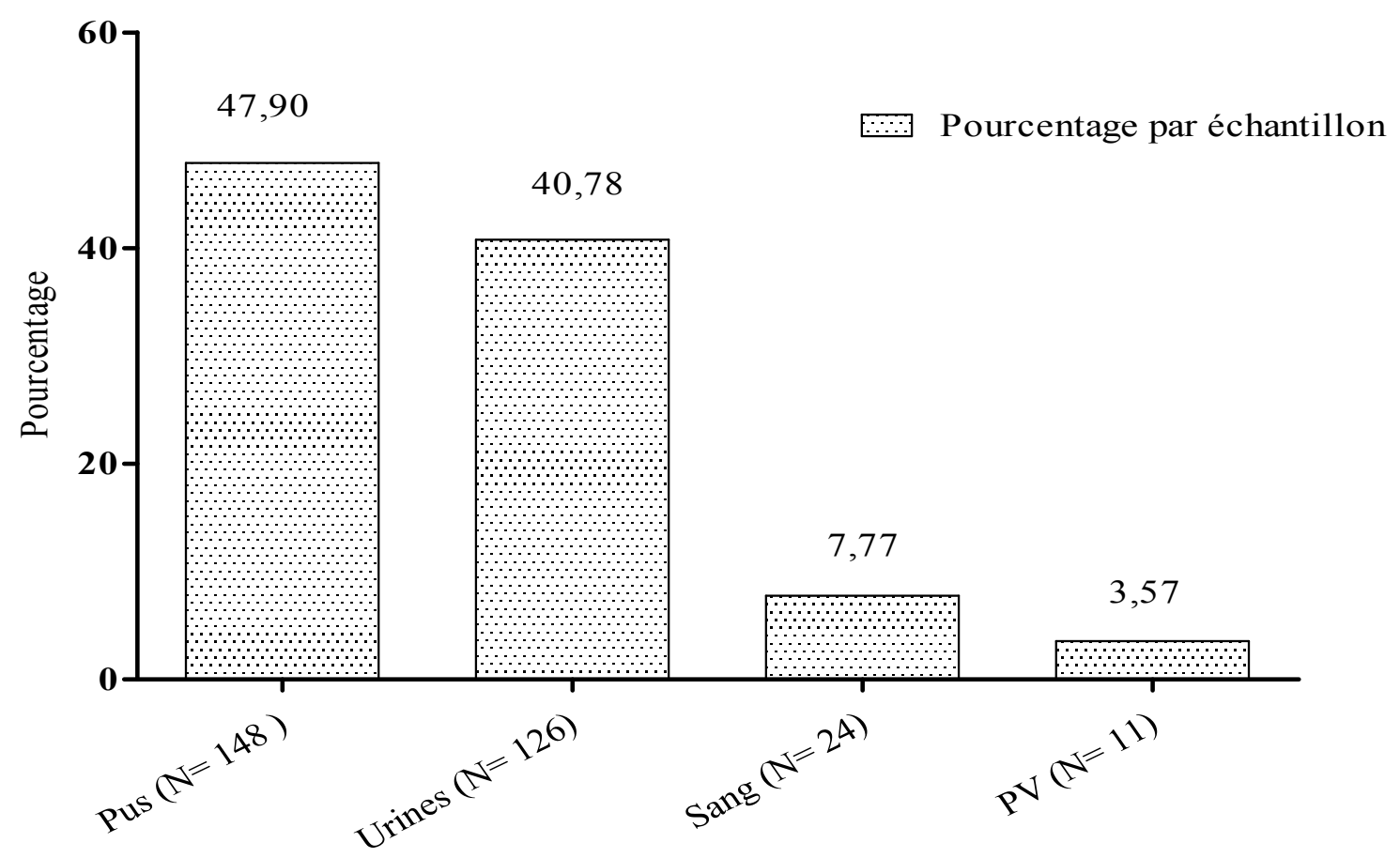

Figure 1: Prévalence des souches d'entérobactéries BLSE par nature du prélèvement

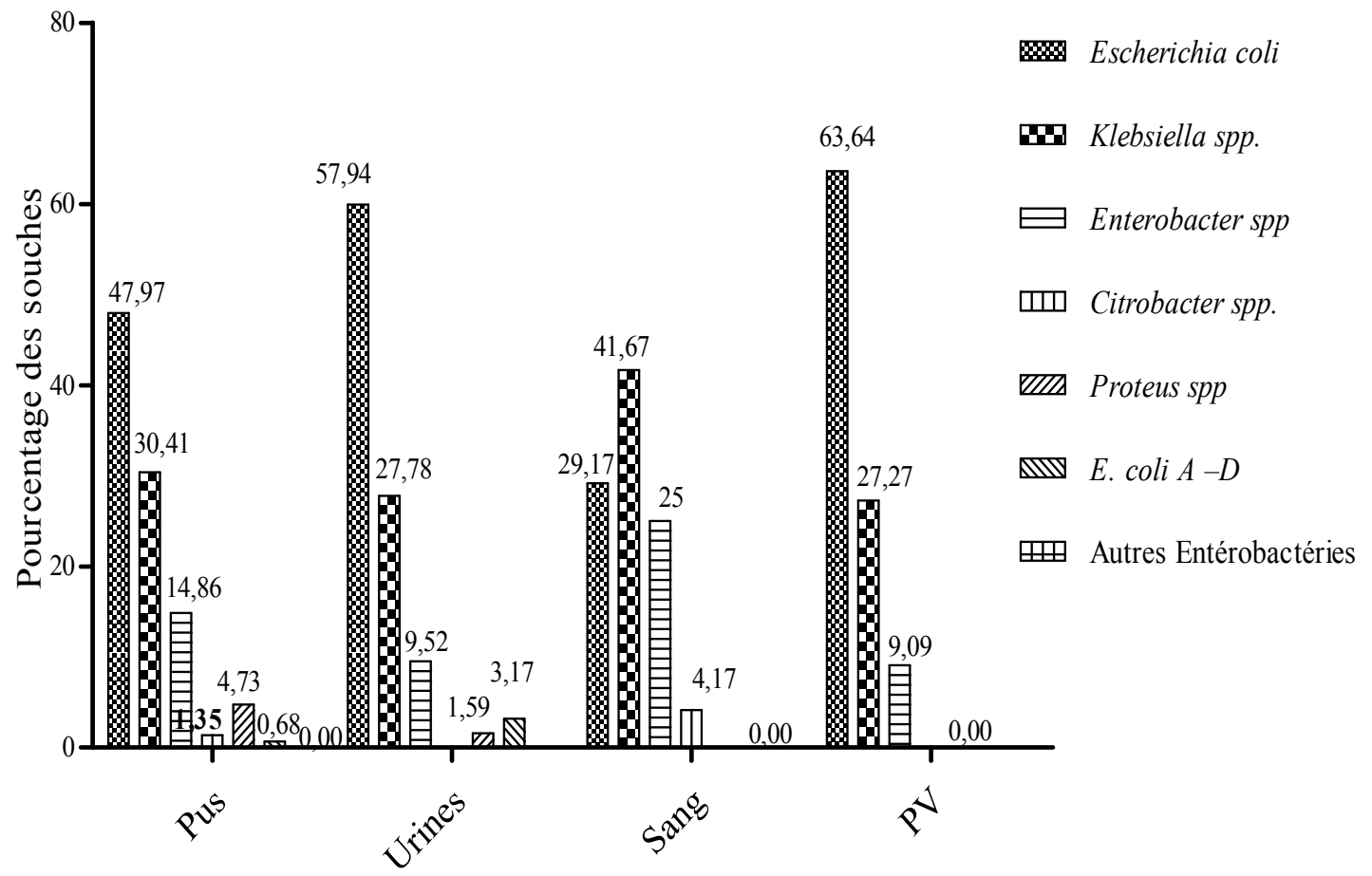

Figure 2: Distribution des souches d'entérobactéries BLSE en fonction du prélèvement 
Tableau 3: Pourcentage de résistance des entérobactéries les plus isolées vis-à-vis des antibiotiques.

\begin{tabular}{|c|c|c|c|c|c|c|c|}
\hline $\begin{array}{l}\text { Classes } \\
\text { d'antisbiotiques }\end{array}$ & $\begin{array}{c}\text { Les } \\
\text { antibiotiques }\end{array}$ & $\begin{array}{l}\text { E. coli } \\
N=158\end{array}$ & $\begin{array}{c}\text { Klebsiella } \\
\begin{array}{c}\text { spp } \\
N=81\end{array}\end{array}$ & $\begin{array}{l}E . s p p \\
N=41\end{array}$ & $\begin{array}{c}\text { E. coli } \\
\begin{array}{c}A-D \\
N=5\end{array}\end{array}$ & $\begin{array}{c}\text { Proteus } \\
\text { Spp. } \\
N=09\end{array}$ & $\begin{array}{c}\text { Valeur } \\
p\end{array}$ \\
\hline \multirow[t]{8}{*}{ Bêta-lactamines } & Amoxicilline & 100 & 100 & 100 & 100 & 100 & - \\
\hline & $\begin{array}{l}\text { Amoxicilline } \\
\text { acide } \\
\text { clavulanique }\end{array}$ & 87,76 & 100 & 100 & 100 & 100 & $<0,001$ \\
\hline & Carbénicilline & 99,2 & 100 & 100 & 100 & 100 & $<0,001$ \\
\hline & Imipenème & 0,68 & 3,7 & 0 & 0 & 0 & 0,016 \\
\hline & Céfalotine & 100 & 98,77 & 100 & 100 & 100 & 0,4 \\
\hline & Cefotaxime & 100 & 100 & 100 & 100 & 100 & - \\
\hline & Ceftazidine & 97,28 & 100 & 100 & 100 & 100 & 0,028 \\
\hline & Ceftriazone & 97,16 & 100 & 100 & 100 & 100 & 0,028 \\
\hline \multirow[t]{4}{*}{ Aminosides } & Gentamicine & 82,2 & 94,64 & 96,3 & - & 100 & $<0,001$ \\
\hline & Amikacine & 13,43 & 4,23 & 2,86 & 0 & 30 & $<0,001$ \\
\hline & Netilmicine & 24,82 & 37,33 & 35,14 & 0 & 40 & $<0,001$ \\
\hline & Kanamycine & 88,89 & 69,23 & 80 & - & 100 & $<0,001$ \\
\hline Phénicolés & Chloramphénicol & 55,56 & 56,86 & 100 & 33,33 & 100 & $<0,001$ \\
\hline Polymyxines & Colistine & 1,38 & 1,27 & 0 & 0 & 100 & $<0,001$ \\
\hline $\begin{array}{l}\text { Quinolone et } \\
\text { Fluoro- }\end{array}$ & $\begin{array}{c}\text { Acide } \\
\text { nalidixique }\end{array}$ & 89,61 & 95,83 & - & - & 100 & 0,03 \\
\hline \multirow[t]{5}{*}{ Quinolones } & Ciprofloxacine & 85,11 & 78,95 & 63,89 & 100 & 70 & $<0,001$ \\
\hline & Péfloxacine & 86,86 & 78,95 & 65,71 & 100 & 70 & $<0,001$ \\
\hline & Levoxacine & 85,04 & 73,02 & 60 & 100 & 70 & $<0,001$ \\
\hline & Ofloxacine & 83,94 & 76,47 & 63,64 & 100 & 62,5 & $<0,001$ \\
\hline & Norfloxacine & 86,67 & 75 & 69,23 & 100 & 62,5 & $<0,001$ \\
\hline \multirow[t]{2}{*}{ Cyclines } & Doxycycline & 97,47 & 89,83 & 96,3 & - & 100 & 0,004 \\
\hline & Tétracycline & 95,53 & 91,11 & 92,59 & 100 & 100 & 0,001 \\
\hline $\begin{array}{l}\text { Sulfamides et } \\
\text { associés }\end{array}$ & $\begin{array}{l}\text { Triméthoprime+ } \\
\text { sulfaméthoxazole }\end{array}$ & 98,15 & 98,31 & 94,44 & 100 & 100 & 0,015 \\
\hline
\end{tabular}

Tableau 4: Etudes statistique sur les diamètres d'inhibition de la céfoxitine sur les souches de Escherichia coli et Klebsiella pneumoniae BLSE.

\begin{tabular}{lcccccc}
\hline Les souches & \% S & Minimum & Médiane & Moyenne & Maximum & P value \\
\hline E. coli, $n=46$ & 83 & 12 & 25 & 23,86 & 27 & 0,0324 \\
K. pneumoniae, $n=52$ & 81 & 10 & 22 & 21,69 & 26 & \\
\hline S : sensible & & & &
\end{tabular}




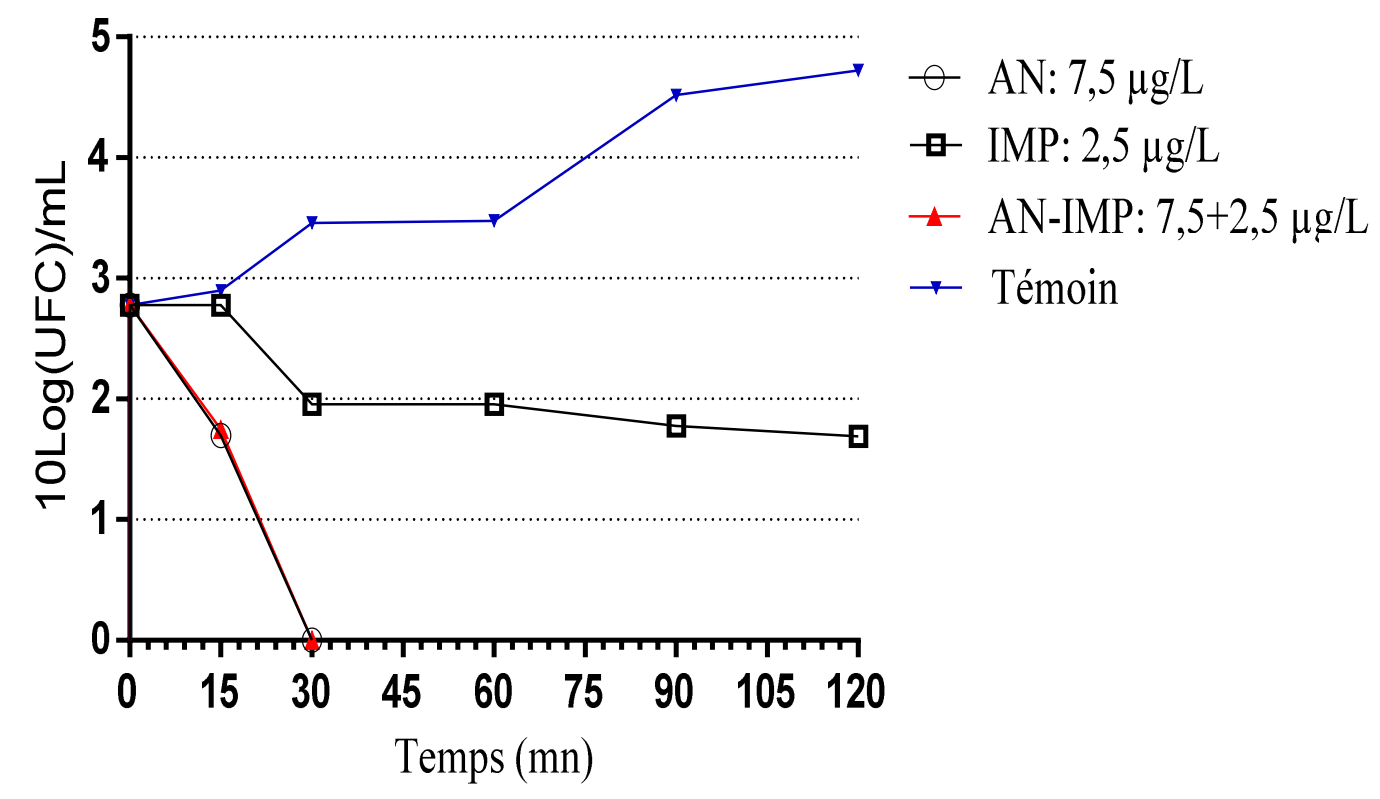

Figure 3: Cinétique de l'activité des associations d'impipénème (IMP) et d'amikacine (AN) vis-àvis des souches de Klebsiella pneumoniae BLSE-.

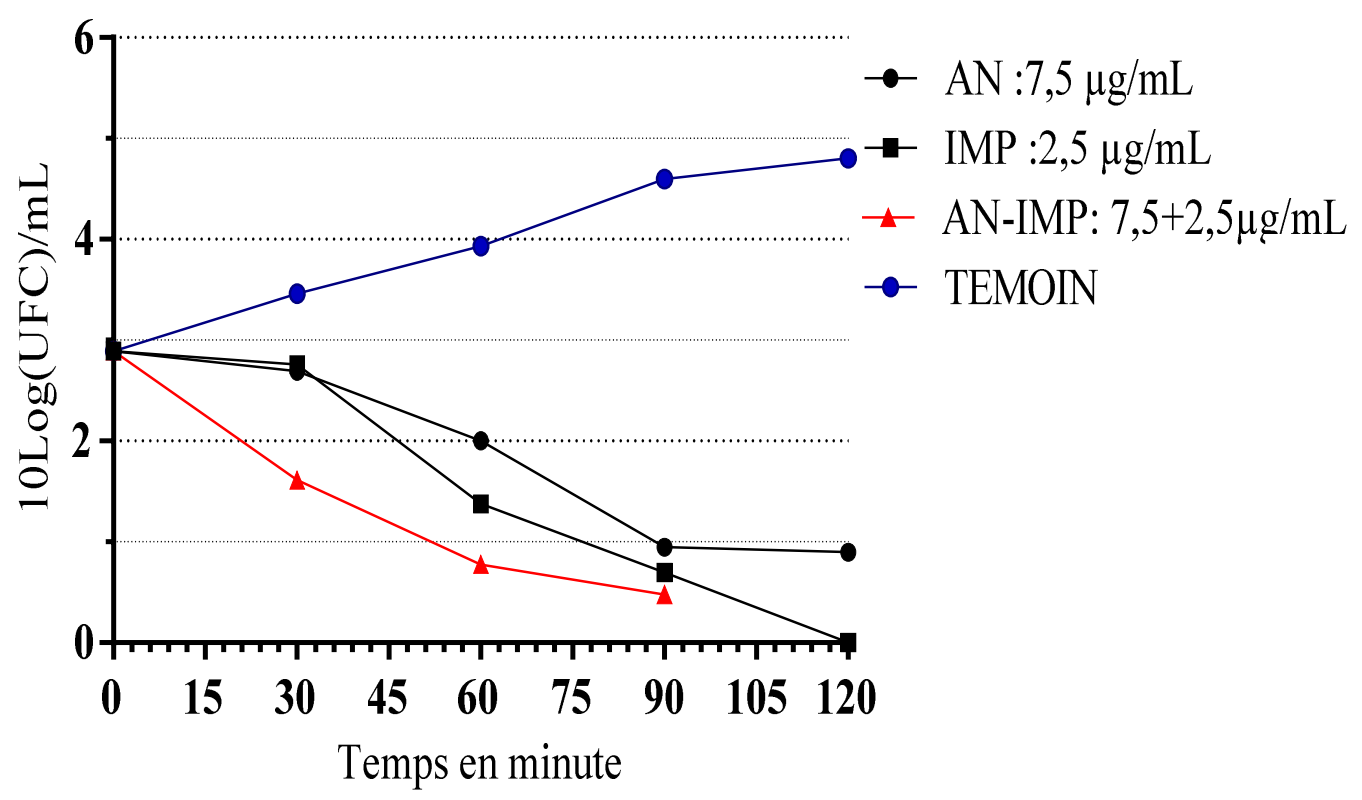

Figure 4: Cinétique de l'activité des associations d'impipénème (IMP) et d'amikacine (AN) vis-àvis des souches de Klebsiella pneumoniae BLSE+. 


\section{DISCUSSION}

Un fait de plus en plus préoccupant ces dernières décennies, dans nos laboratoires de bactériologie médicale, est l'émergence de la multirésistance parmi les souches isolées d'entérobactéries chez les patients. Le problème a été apprécié de plusieurs façons à travers le monde et en Afrique, à l'instar des travaux publiés par Tansarli et al. (2014) sauf au Togo où aucun travail véritable publié ne s'en est clairement chargé. Pour cette raison, notre objectif dans cette étude était d'évaluer la prévalence des entérobactéries productrices de bêta-lactamases à spectre élargi (EBLSE) et leur sensibilité aux antibiotiques. Les souches d'entérobactéries identifiées ont été : E. coli, K. pneumoniae, Enterobacter spp, Citrobacter diversus, Citrobacter freundii, Proteus mirabilis, Proteus vulgaris, Klebsiella oxytoca, E. coli A-D, Morganella morganii. Nos résultats n'ont signalé que quelques genres d'entérobactéries ; ceci correspondant aux travaux effectués au Cameroun par Gangoué-Piéboji et al. (2005) où les genres Salmonella, shigella et yersinia n'ont pas été retrouvés vu leur rareté d'isolement dans les échantillons analysés. Par contre, au Nigéria, Yusha'u et al. (2011) avaient signalé un faible taux de Salmonella Typhi à 2,04\%. La prévalence des genres identifiés était de $22,44 \%$. Cependant, des chiffres supérieurs ont été rapportés dans quelques pays africains au Nigéria à 29\% (Yusha'u et al., 2011); au Sénégal à 1'hôpital de Dakar à 34\% (Fall et al., 2010) et les rapports de Tansarli et al. (2014) ont indiqué des valeurs supérieures à $75 \%$ en Egypte. Par contre, des valeurs bien inférieures ont été trouvées au Cameroun à $12 \%$ (Gangoué-Piéboji et al., 2005) et au Brésil à 7.6\% par Abreu et al. (2013).

La prévalence a varié en fonction des espèces. Elle a été plus forte à $35,34 \%$ chez Enterobacter spp., suivie de Klebsiella spp. à $27,84 \%$ et de E. coli à $23,76 \%$. Parmi les souches productrices de BLSE, la fréquence d'isolement de Enterobacter spp. a été de $13,27 \%$ contre $51,13 \%$ de souches $E$. coli et $30,10 \%$ pour les souches de Klebsiella spp. Ces deux dernières ont occupé $81,23 \%$ du total des souches productrices de BLSE. Plusieurs travaux ont également souligné l'émergence observée au niveau de ces deux souches (Kader et Kumar, 2005 ; Al Jasser, 2006 ; Aminzadeh et al., 2007 ; Zarakolu et al., 2007; Tandé et al., 2009). Une action de lutte privilégierait plus le genre Escherichiae et Klebsiellae. Les pus ont regorgé plus d'EBLSE que les autres prélèvements. Nos résultats corroborent ceux trouvés au Népal (Jeny et Nabaraj, 2015) dans lesquels les entérobactéries ont été majoritairement isolées des pus suivis des urines. Un résultat contraire a été obtenu dans une étude faite en Arabie Saoudite (Kader et Kumar, 2005) où les urines ont contenu plus de BLSE que les suppurations. Les échantillons de sang ont renfermé $7,77 \%$ de EBLSE une valeur similaire à celle rapportée par Jeny et Nabaraj en (2015). La prévalence de $E$. coli a été plus forte dans le PV $(63,64 \%)$ malgré que ce fut l'échantillon ayant regorgé moins de souches BLSE dans l'ensemble. Ce résultat peut s'expliquer par la promiscuité de l'orifice anal et vaginal, étant donné que ceux sont des bactéries qui colonisent fortement le tube digestif et l'orifice anal. Le milieu vaginal élimine physiologiquement par son acidité tous les microorganismes; ces données s'expliquent par le fait que les EBLSE auraient développé un mécanisme pour échapper à ce système de défense. Cette souche a fortement prédominé également dans les urines $(57,94 \%)$ et les pus $(47,97 \%)$. Klebsiella spp. a été prédominante dans les échantillons sanguins $(41,67 \%)$ mais était venu en deuxième position dans les trois autres échantillons. Olonitola et al. (2007) à Zaria au Nigéria avaient prouvé le rôle des souches de E. coli et de Klebsiella pneumoniae dans les infections urinaires, bien entendu que partout dans le monde, la colonisation des urines devient un problème de santé publique majeur (Hailaji et al., 2016; Abreu et al., 2013). En dehors de la sphère périnéale, la sphère respiratoire serait également colonisée par ces souches BLSE (Jeny et Nabaraj, 2015; Olugbue et Onuoha, 2011) et même retrouvées dans les denrées 
alimentaires au Bénin suivant les travaux d'Ahoyo et al. (2010) jusqu'à 35\% de E. coli BLSE.

Les phénotypes de résistance de l'ensemble des entérobactéries étudiés ont été très diversifiés en fonction des classes d'antibiotique (Tableau 3). Ainsi, en dehors de leur forte résistance envers les bêtalactamines, il y a eu des résistances envers les autres familles d'antibiotiques à l'instar des aminosides, des cyclines et des quinolones. Spanu et al. (2002) l'avaient expliqué dans leur travaux en Italie sur la caractérisation des souches d'entérobactéries BLSE. Les EBLE sont donc des souches multirésistantes. Ce fait confirme un choix thérapeutique extrêmement limité pour les patients infectés. L'une des causes serait l'utilisation abusive des antibiotiques démontrée par Trivalle et al. (2006) dans leur étude sur l'écologie des germes isolés chez les personnes âgées hospitalisées. Les bêta-lactamases produites par ces souches leur ont inactivé toutes les pénicillines à un taux compris entre 87,76 et $100 \%$. Toutes les souches étudiées ont été sensibles à $100 \%$ à l'imipénème excepté les souches de E. coli et de K. pneumoniae qui ont eu une résistance bien faible respectivement à $0,68 \%$ et à $3,6 \%$. Une telle observation a été faite sur les souches d'E. coli BLSE dans le département de Zou et Collines au Bénin en 2007 où 5\% avaient résisté à l'imipénème.

Les souches d'E. coli ont été sensibles à $83 \%$ à la céfoxitine, et les $K$. pneumoniae l'ont été à $81 \%$. Ce résultat se rapproche des études de Spanu et al. (2002) en Italie où environ $80 \%$ des souches d'entérobactéries BLSE étaient sensibles à la céfoxitine. La molécule présente sur le noyau bêta-lactame (un radical méthoxyl-0-CH3) protégerait la céfoxitine et l'imipénème contre l'action des enzymes. Selon Singleton et al. (2005), une molécule est protégée de l'action des enzymes par des processus biochimiques tels que la méthylation qui fait greffer un radical méthyle sur le noyau bêta-lactame. C'est ainsi que par exemple, chez beaucoup de bactéries, la réplication de l'ADN est suivie par sa modification c'est-à-dire la méthylation de certaines bases dans des séquences spécifiques de nucléotidique dans chacun des brins nouvellement synthétisés. La méthylation protège l'ADN des endonucléases de restriction. Ici, le noyau bêta-lactame serait protégé par les radicaux méthoxyles, ce qui aurait permis à ces molécules d'antibiotique d'échapper à l'action des bêta-lactamases. La faible résistance observée serait due probablement à la surexpression de céphalosporinase chromosomique ; phénomène plus observé chez les Klebsiella comme souligné dans les travaux de Stewards et al. (2001). La résistance des entérobactéries BLSE aux aminosides était moins élevée que celle observée au niveau des bêta-lactamines. Selon certains auteurs, l'usage abusif des antibiotiques (sous dosage et durée écourtée de traitement) aboutit à la sélection des bactéries résistantes qui, n'ayant pas été éliminées, apprennent à développer des mécanismes de défense de toute sorte y compris la production d'enzymes (Singleton et al., 2005). Ces antibiotiques ne courent pas les rues comme les bêta-lactamines. Leur toxicité est parfois plus remarquable et il n'existe que des formes injectables ce qui limite l'automédication. Par contre, les phénicolés et les fluoroquinolones abondent dans les marchés, disponibles en formes galéniques gélules et comprimés beaucoup prescrits par le corps soignant dans toutes les formes d'infections (Rastogi et al., 2012). Ajoutons aussi que les souches d'E. coli et de Klebsiella spp qui ne produisent pas de bêtalactamases sont naturellement sensibles aux aminosides, aux quinolones; ceux-ci sont des souches de type sauvage. Dans l'étude que nous venons d'effectuer, toutes ces souches ont présenté un taux important de résistance: E. coli de $13,43 \%$ à $88,89 \%$ aux aminosides, $83,94 \%$ à $89,61 \%$ aux quinolones. Cette association de résistance aux quinolones a été prouvée dans les travaux d'Ahoyo et al. (2007) au Bénin. Klebsiella spp a aussi affiché une résistance de 4,23\% à 94,64\% aux aminosides, $75 \%$ à $95,83 \%$ aux quinolones. Ces observations corroborent les travaux de 
Tolun et al. (2004). Selon certains auteurs, le plasmide qui porte le gène de résistance aux bêta-lactamines porterait également le gène QnrA de résistance aux quinolones (Bradford, 2001 ; Paterson et Bonomo, 2005); ce fait expliquerait bien mieux la multirésistance de certaines souches EBLSE. Tous ces résultats ont montré l'effectivité de la présence et la diversité des entérobactéries productrices de BLSE comme l'ont souligné les travaux d'Al Jasser et al. (2006) à travers le monde. Les genres Shigella, Salmonella, et Yersinia désignés "autres entérobactéries » n'ont pas produit de BLSE dans cette étude. Notons que c'est un phénomène très rarement observé en leur sein; mais des travaux récents sur le genre Salmonella producteur de bêtalactamases à spectre élargi isolé dans les échantillons de selles se sont déroulés en 2012 au Népal (Jeny et Nabaraj, 2015) sur une tranche d'âge de 2-60 ans et selon ces résultats, la prévalence a été forte autour de 63\% (Gautam et al., 2012).

\section{Conclusion}

Les études menées dans les établissements de soins nous ont révélé la présence effective d'entérobactéries productrices de bêta-lactamases à spectre élargi à $22,44 \%$. E. coli et Klebsiella spp les plus isolés des infections urinaires et les pus ont représenté successivement $23,76 \%$ et $27,87 \%$ et ont représenté jusqu'à $81,23 \%$ de l'ensemble des EBLSE. Ces souches ont fortement résisté aux bêta-lactamines ainsi qu'à d'autres antibiotiques, ce qui compliquerait sans doute le traitement des patients. La caractérisation moléculaire nous permettrait d'apprécier les différents types de bêta-lactamases circulant.

\section{CONFLITS D'INTERETS}

Les auteurs déclarent qu'il n'y a aucun conflit d'intérêts.

\section{CONTRIBUTIONS DES AUTEURS}

Tous les auteurs ont contribué à la conduite de ce travail. Les auteurs de cet article ont lu et approuvé son contenu.

\section{REFERENCES}

Abreu AG, Marques SG, Monteiro-Neto V, Gonçalves AG. 2013. Extendedspectrum beta-lactamase producing Enterobacteriaceae in communityacquired urinary tract infections in São Luís, Brazil. Braz. J. Microbiol., 44(2): 469-471.

Ahoyo AT, Baba-Moussa L, Anago AE, Avogbe P, Missihoun TD, Loko F, Prévost G, Sanni A, DramaneK. 2007. Incidence d'infections liées à Escherichia coli producteur de bêta lactamases à spectre élargi au Centre hospitalier départemental du Zou et Collines au Bénin. Méd. Mal. Infect., 37(11): 746-752.

Ahoyo TA, Ahissou H, Kounon F, Aminou T, Dramane K. 2010. Etude de la qualité bactériologique des aliments vendus sur le campus de l'Université d'Abomey Calavi au Bénin. Int. J. Biol. Chem. Sci., 4(4): 1083-1092.

Al-Jasser MA. 2006. Extended-Spectrum Beta-Lactamases (ESBLs): A Global Problem. Department of Microbiology, Armed Forces Hospital, Riyadh, Saudi Arabia. Kuwait Med. J., 38(3): 171-185.

Aminzadeh Z, Sadat-Kashi M, Sha'bani M. 2008. Bacteriuria by extended-spectrum beta-lactamase-producing Escherichia coli and Klebsiella pneumoniae: isolates in a governmental hospital in South of Tehran. Iran J. Kidney Dis., 2:197-200.

Bradford PA. 2001. Extended-spectrum ßlactamases in the 21st century. Characterization, epidemiology, and detection of this important resistance threat. Clin. Microbiol. Rev., 14: 933951.

CA-SFM. 2004. Le Rémic: Référentiel en Microbiologie médicale (bactériologie et mycologie. Montmorency: 2M2 (2 $2^{\text {ème }}$ édition). CA-SFM.

Clinical and Laboratory Standards Institute. 2008. Performance standards for Antimicrobial Disk Susceptibility Tests. Approved Standard-Nineteenth edition. CLSI document M100-A19. Clinical and 
Laboratory Standards Institute: Wayne, PA.

Dagnra AY, Tigossou S, Prince-David M. 2000. Prevalence and antimicrobial susceptibility of bacterial meningitis. Med. Mal. Infect., 30: 291-294.

Fall B, Nadiele LP, Ndiaye KS, Diawara S, Ndiaye M, Wade B. Les infections nosocomiales à l'hôpital principal de Dakar: bilan des enquêtes «1 jour donné» de 2006 à 2010 www.icpic2011.com consulté le 18 Août 2011.

Gangoué-Piéboji J, Bedenic B, Koulla-Shiro $\mathrm{S}$, Randegger C, Adiogo D, Ngassam P, Peter Ndumbe P, Hächler H. 2005. Extended-spectrum- $\beta$-lactamase-

producing Enterobacteriaceae in Yaounde, Cameroon. J. Clin. Microbiol., 43(7): 3273-3277.

Gautam K, Pokhrel BM, Bhatta DR, Shrestha CD. 2012. Studies on Extended Spectrum Beta Lactamase (ESBL) producing Salmonella isolates from clinical samples of Nepal. Nepal Med. Coll. J., 14(3): 204-206.

Hailaji NSM, Ould Salem ML, Ghaber SM. 2016. La sensibilité aux antibiotiques des bactéries uropathogènes dans la ville de Nouakchott-Mauritanie. Progrès en urologie, 26: 346-352. http://dx.doi.org/10.1016/j.purol.2016.04 .004 .

Irajian G, Azayeri-Moghadas A, Beheshti A. 2009. Prevalence of extended-spectrum beta lactamase positive and multidrug resistance pattern of Escherichia coli and Klebsiella pneumoniae isolates. Iran J. Microbiol., 1: 49-53.

Jeny P, Nabaraj P. 2015. Multidrug Resistant and Extended Spectrum beta-Lactamase (ESBL) Isolates from different clinical specimens Int. J.Sci. Res. Pub., 5(9):1519.

Kader AA, Kumar A. 2005. Prevalence and antimicrobial susceptibility of extendedspectrum beta-lactamase-producing Escherichia coli and Klebsiella pneumoniae in a general hospital. Ann. Saudi Med., 25(3): 239-242.

Karou DS, Balaka A, Bamoké M, Tchelougou D, Assih M, Anani K, Agbonoko K, Simpore J, de Souza C. 2012. Epidemiology and antibiotic resistance of bacterial meningitis in Dapaong, northern Togo. Asian Pac. J. Trop. Med., 2012: 848-852.

Luzzaro F, Mezzatesta M, Mugnaioli C, Perilli M , Stefani S, Amicosante G, Rossolini GM, Toniolo A. 2006. Trends in Production of Extended-Spectrum $\beta$ Lactamases among Enterobacteria of Medical Interest: Report of the Second Italian Nationwide Survey J. Clin. Microbiol., 44(5):1659-1664.

Olonitola OS, Olayinka AT, Inabo IH, Shaibu AM. 2007. Production of extended spectrum beta-lactamases of urinary isolates of Escherichia coli and Klebsiella pneumoniae in Ahmadu Bello University Teaching Hospital, Zaria, Nigeria. Int. J. Biol. Chem. Sci., 1(2): 181-185.

Olugbue V, Onuoha S. 2011. Prevalence and antibiotic sensitivity of bacterial agents involved in lower respiratory tract infections Int $J$ Biol Chem Sci., 5(2): 774-781.

Paterson DL, Bonomo RA. 2005. Extendedspectrum beta-lactamases: a clinical update. Clin. Microbiol. Rev., 18: 657686.

Quincampoix JC, Mainardi JL. 2001. Mécanismes de résistance des cocci à Gram positif. Réanimation, 10(3): 267275.

Rastogi V, Mishra PK, Bhatia S. 2012. Emerging antimicrobial resistance in hospital a threat to public health. Indian J. Commun. Health, 24(4): 260-263.

Rawat D, Nair D. 2010. Extended-spectrum $\beta$ lactamases in Gram Negative Bacteria. $J$. Glob. Infect. Dis., 2(3): 263-274.

Singleton P. 2005. Bactériologie pour la Médecine, la Biologie et les Biotechnologies ( $6^{\text {ème }}$ édn). (Traduit de l'anglais par Jean Dusart), Dunod, Paris. 
Somily AM, Habib HA, Absar MM, Arshad MZ, Manneh K, Al Subaie SS, Al Hedaithy MA, Sayyed SB, Shakoor Z, Murray TS. 2014. ESBL-producing Escherichia coli and Klebsiella pneumoniae at a tertiary care hospital in Saudi Arabia. J. Infect. Dev. Ctries., 8(9):1129-1136.

Spanu T, Luzzaro F, Perilli M, Amicosante G, Toniolo A, Fadda G. 2002. Occurrence of Extended-Spectrum $\beta$-Lactamases in Members of the Family Enterobacteriaceae in Italy: Implications for Resistance to $\beta$-Lactams and Other Antimicrobial Drugs. Antimicrob. Agents Chemother., 46(1): 196-202.

Stewart CD, Rasheed JK, Hubert SK, Biddle JW, Raney PM, Anderson GJ, Williams PP, Brittain KL, Oliver A, Mcgowan JE, Tenover FC. 2001. Characterization of clinical isolates of Klebsiella pneumonia from 19 laboratories using the National Committee for Clinical Laboratory Standards Extended-Spectrum $ß$ lactamase detection methods. J. Clin. Microbiol., 39: 2864-2872.

Tandé D, Jallot N, Bougoudogo F, Montagnon T, Gouriou S, Sizun J. 2009. Extendedspectrum $\quad \beta$-lactamase producing Enterobacteriaceae in Malian Orphanage. Emerg. Infect. Dis., 15(3): 472-474.

Tansarli GS, Poulikakos P, Kapaskelis A, Falagas ME. 2014. Proportion of extended-spectrum b-lactamase (ESBL)producing isolates among
Enterobacteriaceae in Africa: evaluation of the evidence-systematic review. $J$. Antimicrob. Chemother., 69: 1177-1184.

Tolun V, Küçükbasmaci Ö, TörümküneyAkbulut D, Catal C, Ang-Küçüker M, Ang Ö. 2004. Relationship between ciprofloxacin resistance and extendedspectrum beta-lactamase production in Escherichia coli and Klebsiella pneumonia strains. Clin. Microbiol. Infect.; 10: 72-75

Trivalle C, Lopez Tourres F, Minozzi C, Mathieu D. 2006. Bacterialecology of urine isolates from elderly hospitalized patients: intensive to empirical therapy. Rev. Geriatr., 31(2): 77-82.

Wani KA, Thakur MA, Siraj Fayaz A, Fomdia B, Gulnaz B, Maroof P. 2009. Extended Spectrum B-Lactamase Mediated Resistance in Escherichia Coli in a Tertiary Care Hospital. International Journal of Health Sciences, Qassim University, 3(2): 155-163.

Yusha'u M, Adakawa MI, Kawo AH. 2011. Prevalence of extended spectrum $\beta$ lactamases (ESBLs) among clinical Enterobacteriaceae isolates obtained from private diagnostic laboratory in Kano - Nigeria. Int. J. Biol. Chem. Sci., 5(1): 365-370.

Zarakolu P, Metan G, Hasçelik G, Akova M. 2007. Prevalence of extended-spectrum beta-lactamases in nosocomial Escherichia coli and Klebsiella spp. strains isolated from blood cultures. Mikrobiyol Bul., 41(4): 579-584. 